\title{
TIGHTNESS IN PRODUCT SPACES
}

\author{
U. N. B. DISSANAYAKE AND S. W. WILLARD ${ }^{1}$
}

\begin{abstract}
A product $\prod X_{i}$ of topological spaces $X_{i}, i \in I$, will be said to preserve tightness if

$$
\partial\left(\prod X_{i}\right) \leq|I| \cdot \sup \left\{\partial\left(X_{i}\right) \mid i \in I\right\}
$$

where $\partial(X)$ denotes the tightness of $X$.

We show $\prod X_{i}$ preserves tightness whenever each finite subproduct does. It is further shown that this is the case whenever each $X_{i}$ is a locally compact $T_{2}$-space, and whenever each $X_{i}$ is a locally Lindelöf $T_{3} P$-space, extending 5.9 in $[\mathbf{J}]$.
\end{abstract}

Preliminaries. Let $m$ and $n$ be infinite cardinals, $m \geq n$. A space $X$ is said to be $m-n$ compact iff every open cover $\mathcal{U}$ of $X$ of cardinality $\leq m$ has a subcover of cardinality $<n$. If every open cover of $X$ admits a subcover of cardinality $<n$, we call $X \infty-n$ compact. These spaces have been considered in $[\mathbf{G}, \mathbf{N}$ and $\mathbf{V}]$. Our notation follows that in $[\mathbf{N}]$.

A filter $\mathcal{F}$ on $X$ is an $m$-n filter provided it has a filter base $\mathcal{F}_{0}$ with cardinality $\leq m$ and the intersection of any subfamily of cardinality $<n$ is nonvoid (i.e. $\mathcal{F}$ has the $<n$-intersection property).

A space $X$ is $<n$-discrete provided every intersection of fewer than $n$ open sets is open.

We quote the following standard results for future reference.

1.1 TheOREM (GAL [G, TheOREM 1.1]). A space $X$ is $m$ - $n$ compact iff every $m-n$ filter on $X$ has a cluster point.

1.2 THEOREM (KUNEN). Every $\infty$ - $n$ compact, $<n$-discrete $T_{3}$-space is paracompact.

1.3 TheOREM (VAughan [V, TheOREM 3.2]). Let $n$ be a regular cardinal. If $X$ is $\infty$ - $n$ compact and $<n$-discrete for $i=1,2, \ldots$, then $\prod_{i=1}^{\infty} X_{i}$ is $\infty$ - $n$ compact.

The following result will also be needed in the sequel. It can easily be derived as a consequence of Theorem 2.2 in $[\mathbf{N}]$; a direct proof is not difficult and will be provided here.

1.4 THEOREM. If $X$ is $<n$-discrete and has character $\leq m$, and $Y$ is $m-n$ compact, the projection $\pi_{X}: X \times Y \rightarrow X$ is closed.

Received by the editors October 17, 1983 and, in revised form, February 4, 1985.

1980 Mathematics Subject Classification. Primary 54A25, 54B10, 54D30.

Key words and phrases. Tightness, product space, $m$ - $n$ compact space, $<n$-discrete space.

${ }^{1}$ Supported in part by Grant \#A-7591 from the National Science and Engineering Research Council of Canada. 
Proof. Let $F \subset X \times Y$ be closed, $x \in \overline{\pi_{x}(F)}$. Let $\nu_{X}$ be a neighbourhood system at $x$ in $X$ with $\left|\nu_{X}\right| \leq m$. Set

$$
\mathcal{G}=\left\{\pi_{X}^{-1}(V) \cap F \mid V \in \nu_{X}\right\}
$$

and let $\pi_{Y}(\mathcal{G})=\left\{\pi_{Y}(G) \mid G \in \mathcal{G}\right\}$. Then easily $\pi_{Y}(\mathcal{G})$ is a base for an $m$ - $n$ filter on $Y$, and hence by 1.1 , we can find some $y \in\left\{\bigcap\left\{\overline{\pi_{Y}(G)}\right\} \mid G \in \mathcal{G}\right\}$.

Now if $V$ and $W$ are neighbourhoods of $x$ and $y, W$ meets $\pi_{Y}\left[\pi_{X}^{-1}(V) \cap F\right]$ and hence $\pi_{Y}^{-1}(W) \cap \pi_{X}^{-1}(V) \cap F \neq \varnothing$. Thus every (basic) neighbourhood $V \times W$ of $(x, y)$ in $X \times Y$ meets $F$, so $(x, y) \in \bar{F}=F$, and hence $x \in \pi_{X}(F)$.

Finally, we introduce some new terminology. A space $X$ will be called locally $\infty-n$ compact if each $x \in X$ has a base of $\infty-n$ compact neighbourhoods. We will call $X$ a $\mathrm{GC}(n, i)$-space, for $i=1,2,3,4$, if $X$ is an $\infty$ - $n$ compact, $<n$-discrete $T_{i}$ space, and $X$ will be a $\operatorname{GLC}(n, i)$-space, for $i=1,2,3,4$, if $X$ is a locally $\infty-n$ compact, $<n$-discrete $T_{i}$ space.

2. Tightness in small products. We denote the tightness (cf. Juhasz $[\mathbf{J}$, 1.16]) of a topological space $X$ by $\partial(X)$. Recall that a subset $F$ of $X$ is $\kappa$-closed, where $\kappa$ is an infinite cardinal iff whenever $S \subset F$ and $|S| \leq \kappa$ then $\bar{S} \subset F$.

The following facts are standard and will be used in the sequel without specific reference:

(a) Every closed set in $X$ is $\kappa$-closed.

(b) Any intersection of $\kappa$-closed sets is $\kappa$-closed.

(c) If $f$ is a closed map of $X$ onto $Y$ and $F \subset X$ is $\kappa$-closed in $X$, then $f(F)$ is $\kappa$-closed in $Y$.

(d) $\partial(X) \leq \kappa$ iff every $\kappa$-closed set in $X$ is closed.

The results of this section will stem from the following basic result. The proof mimics in detail the proof given in [J, p. 113] for the case $n=\aleph_{0}$.

2.1 THEOREM. If $X$ is $a<n$-discrete $T_{1}$-space and $Y$ is an $\infty$ - $n$ compact regular space, then

$$
\partial(X \times Y) \leq \partial(X) \cdot \partial(Y)
$$

ProOF. Let $\kappa=\partial(X) \cdot \partial(Y)$ and suppose $H \subset X \times Y$ is $\kappa$-closed. It suffices to show $H$ is closed.

Let $(p, q) \in H$. If $T=H \cap(\{p\} \times Y)$, then $T$ is $\kappa$-closed and hence closed in $\{p\} \times Y$, and hence closed in $X \times Y$. We need only show $q \in \pi_{Y}(T)$.

Suppose $q \in \pi_{Y}(T)$. Since $\pi_{Y} \mid\{p\} \times Y$ is a homeomorphism, $\pi_{Y}(T)$ is closed in $Y$. Let $V$ be a closed neighbourhood of $q$ such that $V \cap \pi_{Y}(T)=\varnothing$. Note that since $X \times V$ is a neighbourhood of $(p, q)$ and $(p, q) \in \bar{H}$, we have $(p, q) \in \overline{(X \times V) \cap H}$. But $(X \times V) \cap H$ is a closed subset of $H$, and hence $\kappa$-closed in $X \times Y$. Since $Y$ is $\infty$ - $n$ compact and $X$ is $<n$-discrete, $\pi_{X}$ is closed (by 1.4). Thus $\pi_{X}[(X \times V) \cap H]$ is $\kappa$-closed and hence closed in $X$. Then, since $\pi_{X}$ is continuous,

$$
p \in \pi_{X} \overline{[(X \times V) \cap H]} \subset \overline{\pi_{X}[(X \times V) \cap H]}=\pi_{X}[(X \times V) \cap H] .
$$

So for some $v \in V,(p, v) \in H$. But then $v \in \pi_{Y}(T) \cap V$, a contradiction.

2.2 THEOREM. If $X$ is $<n$-discrete $T_{1}$ and $Y$ is locally $\infty$ - $n$ compact $T_{3}$, then $\partial(X \times Y) \leq \partial(X) \partial(Y)$.

Proof. Let $K=\partial(X) \cdot \partial(Y)$ and let $H \subset X \times Y$ be $\kappa$-closed. Choose $(p, q) \in \bar{H}$. Let $V$ be any closed $\infty-n$ compact neighbourhood of $q$. Then $X \times V$ is closed in 
$X \times Y$ and $\partial(X \times V) \leq \kappa$ by 2.1. But $H \cap(X \times V)$ is $\kappa$-closed in $X \times V$, and thus closed in $X \times V$, and $(p, q) \in \mathrm{Cl}_{X \times V} H \cap(X \times V)=H \cap(X \times V)$. Thus $(p, q) \in H$.

2.3 CoROllary. If $X_{1}, X_{2}, \ldots, X_{n}$ are $\operatorname{GLC}(n, 3)$ spaces, then

$$
\partial\left(\prod_{i=1}^{n} X_{i}\right) \leq \partial\left(X_{1}\right) \cdots \partial \partial\left(X_{n}\right) .
$$

PROOF. The proof is by induction, noting that

$$
X_{1} \times \cdots \times X_{k}=\left(X_{1} \times \cdots \times X_{k-1}\right) \times X_{k}
$$

and that $X_{1} \times \cdots \times X_{k-1}$ is $<n$-discrete and $T_{1}$, while $X_{k}$ is locally $\infty$ - $n$ compact and $T_{3}$, so that

$$
\partial\left(X_{1} \times \cdots \times X_{k}\right) \leq \partial\left(X_{1} \times \cdots \times X_{k-1}\right) \cdot \partial\left(X_{k}\right)
$$

by 2.2 .

Thus, finite products of locally compact $T_{2}$ spaces $\left(=\operatorname{GLC}\left(\aleph_{0}, 2\right)\right)$ preserve tightness, as do finite products of locally Lindelöf $T_{3} P$-spaces $\left(=\operatorname{GLC}\left(\aleph_{1}, 3\right)\right)$, and so on.

Infinite products will be considered in the next section; first an example, showing that Theorem 2.2 cannot be improved for $T_{3}$ spaces.

2.4 EXAMPLE. In $[\mathbf{T}]$, an example is given of a Lindelöf space $Y$ and an $\infty-c^{+}$ compact space $X$, each sequential and therefore of countable tightness, whose product has uncountable tightness. Since only an outline of proof is included in the reference, we will develop the example fully here.

The space $Y$, the countable fan, is the quotient obtained by identifying to a single point all nonisolated points in the disjoint union of denumerably many copies of the converging sequence, while $X$, the $c$-fan, is obtained by applying the same process to $c$ copies of the converging sequence. Each is a quotient of a metric space, hence sequential, hence of countable tightness. To show that $X \times Y$ fails to have countable tightness, we will employ a more notational approach to $X$ and $Y$.

Let $X$ and $Y$ be the following subsets of $R \times R$ :

$$
\begin{aligned}
& X=\{(0,0)\} \cup\left\{(r, n) \mid r \in R^{+}, n=1,2, \ldots\right\}, \\
& Y=\{(0,0)\} \cup\{(m, n) \mid m=1,2, \ldots, n=1,2, \ldots\} .
\end{aligned}
$$

All points in $X$ and $Y$ will be isolated except the points $(0,0)$. A typical basic neighbourhood $B$ of $(0,0)$ in $X$ will be obtained by choosing a positive integer $m_{r}$ for each $r \in R^{+}$and setting

$$
B=\{(0,0)\} \cup\left\{(r, n) \mid n>m_{r}\right\},
$$

while a typical basic neighbourhood $C$ of $(0,0)$ in $Y$ will be obtained by choosing a positive integer $n_{m}$ for each $m=1,2, \ldots$ and setting

$$
C=\{(0,0)\} \cup\left\{(m, n) \mid n>n_{m}\right\} .
$$

We claim $X \times Y$ does not have countable tightness; we will, in fact, produce a subset $S$ of $[X-\{(0,0)\}] \times[Y-\{(0,0)\}]$ such that $(0,0,0,0) \in \bar{S}$ in $X \times Y$, but $(0,0,0,0)$ is not in the closure of any countable subset of $S$.

Note that the basic neighbourhood system $C$ at $(0,0)$ in $Y$ has cardinality $c$ and let $f$ be any one-one map of $C$ onto $R^{+}$, denoting $f(C)$ by $r_{C}$ for each $C \in C$. 
For $C \in C$, select a sequence $X_{C 1}, X_{C 2}, \ldots$ in $X \times Y$ by choosing $n_{C m}$ for each $m=1,2, \ldots$ so that $\left(m, n_{C m}\right) \in C-\{0,0\}$ and setting

$$
X_{C m}=\left(r_{C}, m, m, n_{C m}\right) \text {. }
$$

Now set $S=\left\{X_{C m} \mid C \in C, m=1,2, \ldots\right\}$.

We first show $(0,0,0,0) \in \bar{S}$ in $X \times Y$. Let $B \times C$ be a (basic) neighbourhood of $(0,0,0,0)$ in $X \times Y$. Then $B$ includes for some choice(s) of $m$, a point of the form $\left(r_{C}, m\right)$ and then $X_{C m}=\left(r_{C}, m, m, n_{C m}\right)$ belongs to $\left\{\left(r_{C}, m\right)\right\} \times(C-\{(0,0)\})$ and hence to $B \times C$.

Now we claim $(0,0,0,0) \notin \bar{M}$ if $M \subset S$ is countable. The first coordinates of points of $M$ occur, let us say, as $r_{C_{1}}, r_{C_{2}}, \ldots$ We will construct a neighbourhood of $(0,0,0,0)$ in $X \times Y$ which includes no such point of $S$. Choose a (basic) neighbourhood $B$ of $(0,0)$ in $X$ by setting $m_{r_{C_{n}}}=n$ for each $n=1,2, \ldots$ (and letting $m_{r}$ be any positive integer where $r \neq r_{C_{n}}$ for some $n$ ). Choose a (basic) neighbourhood $C$ of $(0,0)$ in $Y$ by setting

$$
n_{l}>\max \left\{n_{C_{1} l}, n_{C_{2} l}, \ldots, n_{C_{l} l}\right\}
$$

for each $l=1,2, \ldots$ Now we claim $B \times C$ contains no point in $S$ with first coordinate $r_{C_{k}}$ for any $k$, that is, no point of the form $X_{C_{k} l}=\left(r_{C_{k}}, l, l, n_{C_{k} l}\right)$. For if $\left(r_{C_{k}}, l\right) \in B$, then $l>k$, and hence $n_{l}>n_{C_{k} l}$ and $\left(l, n_{C_{k} l}\right) \notin C$. Thus $B \times C$ contains no point of $M$.

3. Tightness in large products. If $R=\left\{R_{i} \mid i \in I\right\}$ is any collection of topological spaces, we define

$$
\partial_{I}(R)=\sup \left\{\partial\left(R_{i}\right) \mid i \in I\right\} .
$$

We will say the product $R=\prod_{i \in I} R_{i}$ preserves tightness iff

$$
\partial\left(\prod_{i \in I} R_{i}\right) \leq|I| \cdot \partial_{I}() .
$$

For finitely many spaces $X_{1}, \ldots, X_{n}$ this reduces to the assertion that

$$
\partial\left(\prod_{i=1}^{n} X_{i}\right) \leq \partial\left(X_{1}\right) \cdots \cdot \partial\left(X_{n}\right)
$$

and thus the results of the previous section.

It will be convenient to introduce some notation. Let $R=\prod_{i \in I} R_{i}$ and suppose $J \subset I$. The subproduct $\prod_{i \in J} R_{i}$ of $R$ will be denoted $R_{J}$, and the projection of $R$ onto $R_{J}$ will be denoted $\pi_{J}$. For $a \in R$ and $A \subset R$ we will denote by $a_{J}$ and $A_{J}$ the images $\pi_{J}(a)$ and $\pi_{J}(A)$, respectively.

Our main result is a generalization of a part of Theorem 5.9 in $[\mathbf{J}]$ and is proved in similar fashion. Since it reduces the question of preservation of tightness in a large product to consideration of its small subproducts, our concrete results will be corollaries to results of the previous section.

3.1 THEOREM. If each finite subproduct of a product $R=\prod_{i \in I} R_{i}$ preserves tightness, then $R$ preserves tightness.

ProOF. Let $R=\left\{R_{i} \mid i \in I\right\}$ and set $\kappa=|I| \cdot \partial_{I}(R)$. Let $I^{(F)}=\{J \subset I|| J \mid<$ $\left.\aleph_{0}\right\}$. 
Suppose $A \subset R$ is $\kappa$-closed, and $a \in \bar{A}$. Then for $J \in I^{(F)}, a_{J}$ belongs to $\pi_{J}(\bar{A}) \subset \bar{A}_{J}$. Since $\partial\left(R_{J}\right) \leq \kappa$, we can find $B_{J} \subset A_{J}$ with $\left|B_{J}\right| \leq \kappa$ such that $a_{J} \in \bar{B}_{j}$.

For each $b \in B_{J}$, choose $x_{b} \in A$ so that $\pi_{J}\left(x_{b}\right)=b$, and set $C_{J}=\left\{x_{b} \mid b \in B_{j}\right\}$. Clearly $\left|C_{J}\right|=\left|B_{J}\right| \leq \kappa$ and hence if $C=\bigcup\left\{C_{J} \mid J \in I^{(F)}\right\}$, then $C \subset A$ and $|C| \leq \kappa \cdot|I|=\kappa$.

But $a \in \bar{C}$. For if $U=U_{J} \times \prod_{i \in J} R_{i}$ is a basic open neighbourhood of $a$, then since $\pi_{J}(a) \in \overline{B_{J}}=\overline{\pi_{J}\left(C_{J}\right)}$, we have $U_{J} \cap \pi_{J}\left(C_{J}\right) \neq \varnothing$, and thus $U \cap C_{J} \neq \varnothing$, whence $U \cap C \neq \varnothing$.

Thus $a \in A$, and $A$ is closed.

3.2 COROLlaRY. If $R_{i}$ is a $\operatorname{GLC}(n, 3)$ space for each $i \in I$, then $\prod R_{i}$ preserves tightness.

ProOF. Apply 3.1 and 2.3.

In $[\mathbf{J}, 5.9]$ it is shown that any product of compact $T_{2}$ spaces preserves tightness. The first of the various special cases of 3.2 generalizes this result.

3.3 CoROLlaRY. If $R_{i}$ is locally compact and $T_{2}$, for each $i \in I$, then $\prod R_{i}$ preserves tightness.

ProOF. Locally compact, $T_{2} \equiv \operatorname{GLC}\left(\aleph_{0}, 2\right)$.

3.4 CoRollary. If $R_{i}$ is a locally Lindelöf, $T_{3} P$-space, for each $i \in I$, then $\prod R_{i}$ preserves tightness.

Proof. Locally Lindelöf, $T_{3} P$-space $\equiv \operatorname{GLC}\left(\aleph_{1}, 3\right)$.

\section{REFERENCES}

[G] I. S. Gal, On the theory of $(m, n)$-compact topological spaces, Pacific J. Math 8 (1958), 721-734.

[J] I. Juhasz, Cardinal functions in topology-ten years later, Mathematical Centre Tract 123, Mathematisch Centrum, Amsterdam, 1980.

[N] N. Noble, Products with closed projections, Trans. Amer. Math. Soc. 140 (1969), 381-391.

[T] M. G. Tkacenko, The notion of 0-tightness and $C$-embedded subspaces of products, Topology Appl. 15 (1983), 93-98.

[V] J. E. Vaughan, Product spaces with compactness-like properties, Duke Math. J. 39 (1972), 611-617.

Department of Mathematics, University of Alberta, Edmonton, Alberta, CANADA T6G 2G1 